\title{
Dynamic Modelling and Optimal Control Scheme of Wheel Inverted Pendulum For Mobile Robot Application
}

\author{
Mohamad Amir Shamsudin*1 ${ }^{1}$, Rosbi Mamat* ${ }^{2}$, and Sophan Wahyudi Nawawi* ${ }^{3}$ \\ *Department of Control and Mechatronics, Faculty of Electrical, \\ Universiti Teknologi Malaysia, Johor, Malaysia
}

\begin{abstract}
Unstable wheel inverted pendulum is modelled and controlled deploying Kane's method and optimal partial-state PID control scheme. A correct derivation of nonlinear mathematical model of a wheel inverted pendulum is obtained using a proper definition of the geometric context of active and inertia forces. Then the model is decoupled to two linear subsystems namely balancing and heading subsystems. Afterward partial-state PID controller is proposed and formulated to quadratic optimal regulation tuning method. It enables partial-state PID to be optimally tuned and guarantees a satisfactory level of states error and a realistic utilization of torque energy. Simulation and numerical analyses are carried out to analyse system's stability and to determine the performance of the proposed controller for mobile wheel inverted pendulum application.
\end{abstract}

\section{KEYWORDS}

Wheel inverted pendulum, Kane's method, partial-state PID, quadratic optimal regulation tuning

\section{INTRODUCTION}

The application of wheel inverted pendulum (WIP) robot gains high attention from industry and research communities. Recently, many WIP-based products are available in industry such as human transporter by Karmen and service robot by Hitachi's Mechanical Engineering Research laboratory. They believe this high manoeuvrability wheel system will be the future urban robot and transporter and even in rural places with rough terrain. Meanwhile in research community, WIP system has been extensively studied because this simple structure robot serve complex dynamic [1]. Thus many comprehensive research materials can be found and mainly for modelling and control aspects.

Over a decade, various types of WIP have been developed along with their mathematical model [2-5]. The modelling focuses either on kinematic $[6,7]$ or dynamic issues. In dynamic aspect, Lagrange [8, 9], Lagrage-Eular [10], and Newton-Eular [11] used to develop nonlinear WIP mathematical model. However Newton method involved the calculation of unwanted forces and Lagrange method used Lagrange multiplier in their derivation. Consequently those methods required tedious and complicated formulation of a large multibody system, hence led to inefficient computation. Thus, Kane method is the alternative because of its simpler and efficient mathematical analysis where it is based on partial velocity in multibody dynamic modelling [12]. However, less attention on torque context in term of differential drive nonholonomic and mobile system led to an unaccurate dynamic equation [13]. Thus this paper defines a proper torque 
geometric context of WIP system, eventually this definition leads to a correct solution of the nonlinear WIP mathematical model.

In control aspect, advanced and robust control schemes designed to control WIP system. It were sliding mode control (SMC) [14], backsteping control [15], fuzzy logic control (FLC) [16], and neural network control (NN) [17]. However, most of those control schemes need high computationally effort even for single state control [18]. This prevented it to be programmed as a low level controller in an embedded WIP system. Thus linear controller such as LQR and PID controllers are more favourable for this fast system [19-21]. Many researches preferred and showed that the LQR as a modern controller gave better performance compared to conventional PID controller [22-24]. However the research overlooked the applicability of LQR controller in input trajectory tracking where the significant state trajectory errors occur [25]. Thus this paper proposes partial-state PID (PSPID) controller to eliminate the aforementioned problem. Besides, the controller is formulated to quadratic optimal regulation (QOR) tuning scheme where it guarantees system stability and satisfactory level of WIP performance.

This paper is organized as follows: development of nonlinear WIP mathematical model is presented in section 2; section 3 provides a formulation of proposed PSPID controller for the QOR tuning method; the analysis of the model and the proposed controller is demonstrated by simulation in section 4; and the conclusion of this paper is in the last section.

\section{WHEEL INVERTED PENDULUM ROBOT}

Generally, the outline of Kane's method can be found in following literatures [12, 13]. Thus this paper will only concentrate on the development of a WIP mathematical model of Kane's method. Notation: Throughout this section, $\mathrm{C}_{\mathrm{i}}$ and $\mathrm{S}_{\mathrm{i}}$ indicate $\cos$ and $\sin$ functions with $\mathrm{i}=2=\phi$ and $\mathrm{i}=3=\theta$. Body $\mathrm{B}_{\mathrm{i}}$ and point $\mathrm{P}_{\mathrm{i}}$ refer to bodies $\mathrm{B}, \mathrm{W}_{1}, \mathrm{~W}_{2}$ and points $\mathrm{G}, \mathrm{W}_{\mathrm{C} 1}$, and $\mathrm{W}_{\mathrm{C} 2}$.

\subsection{Kinematic of WIP}

As shown in Fig. 1, WIP consists of three bodies: the pendulum, B, as the main body and the two wheels, $\mathrm{W}_{1}$ and $\mathrm{W}_{2}$. The important points where the gravitational force, $\mathrm{g}$ is detected are the centre of right wheel, $\mathrm{W}_{\mathrm{C} 1}$, the centre of left wheel, $\mathrm{W}_{\mathrm{C} 2}$, and the centre of gravity of the main body $\mathrm{G}$; point $\mathrm{C}$, the point where the torque exerted from wheels to pendulum in the form of force. Other vital points on the right and left wheel's surfaces are $\mathrm{W}_{\mathrm{P} 1}$ and $\mathrm{W}_{\mathrm{P} 2} \cdot \mathrm{W}_{\tilde{\mathrm{P}} 1}$ and $\mathrm{W}_{\tilde{\mathrm{P}} 2}$ are any point on the right and left wheels. WIP mechanical parameters tabulate in Table 1 . The WIP has 5 degrees of freedoms, hence the independent generalized coordinates are

$$
q_{n}=\left(q_{1}, q_{2}, \ldots, q_{n}\right)=\left(x, \phi, \theta, \varphi_{W 1}, \varphi_{W 2}\right) ; n=5,
$$

The following assumptions have been made to model the WIP:

1) The linear motor model considered, motor inductance and friction on the armature are neglected.

2) No slip and wheels always in contact with the ground

3) At every instant, WIP experiences forward and steering forces.

4) Cornering force is neglected 
Based on the $2^{\text {nd }}$ and $3^{\text {rd }}$ assumptions, the constraints $\mathrm{m}=2$ and the nonholonomic constraints are

$$
\begin{aligned}
& { }^{F} \hat{\omega}^{W_{\tilde{P} 1}}-{ }^{F} \hat{\omega}^{W_{P 1 \dot{x}}}-{ }^{F} \hat{\omega}^{W_{P 1 \dot{\phi}}}=0, \\
& { }^{F} \hat{\omega}^{W_{\tilde{P} 2}}-{ }^{F} \hat{\omega}^{W_{P 2 \cdot \dot{x}}}-{ }^{F} \hat{\omega}^{W_{P 2 \dot{\phi}}}=0,
\end{aligned}
$$

Table 1.WIP mechanical parameters.

\begin{tabular}{|c|c|c|}
\hline Symbol & Parameter & Value \\
\hline$m_{b}$ & Mass of body & $15 \mathrm{~kg}$ \\
\hline$m_{w}$ & Mass of wheel & $0.42 \mathrm{~kg}$ \\
\hline$L$ & Half of lateral body & $0.2 \mathrm{~m}$ \\
\hline$R$ & Radius of wheel & $0.106 \mathrm{~m}$ \\
\hline$I_{b 2}$ & Inertia of main body in $\mathbf{n}_{\mathbf{2}}$ directional & $0.63 \mathrm{kgm}^{2}$ \\
\hline$I_{b 3}$ & Inertia of main body in $\mathbf{n}_{\mathbf{3}}$ directional & $1.12 \mathrm{kgm}^{2}$ \\
\hline$g$ & Gravitational constant & $9.81 \mathrm{~ms}^{-2}$ \\
\hline$d$ & A distance between point $C$ and COG of main body & $0.212 \mathrm{~m}$ \\
\hline
\end{tabular}

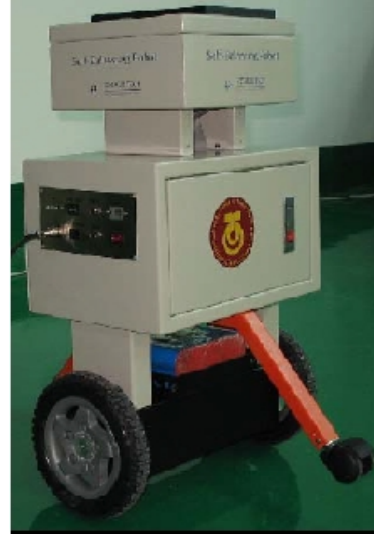

(a)

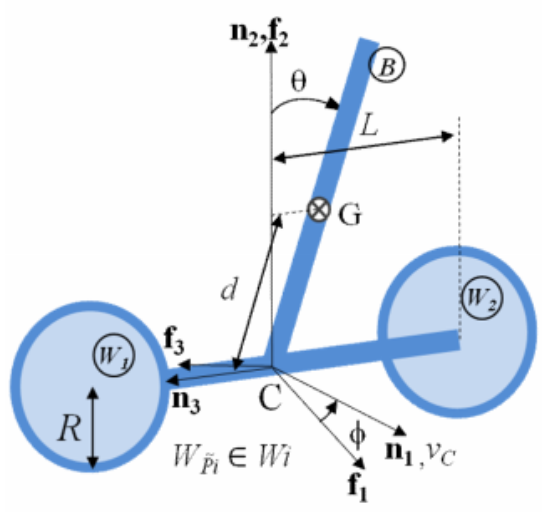

(b)

Figure 1. (a) Available WIP in UTM laboratory, and (b) free body diagram of WIP in newton Frame $F$.

${ }^{F} \hat{\omega}^{W_{\tilde{P} i}}$ is an angular velocity of point $\mathrm{W}_{\tilde{\mathrm{P}} \mathrm{i}}$ on the right and left wheels with respect to reference frame $F .{ }^{F} \hat{\omega}^{W_{P 1 \dot{x}}}$ and ${ }^{F} \hat{\omega}^{W_{P 1 \dot{\phi}}}$ are angular velocities of points $\mathrm{W}_{\mathrm{P} 1} s$ on the right and left wheels with respect to frame $F$ that due to forward motion and steering motion. Based on (1) and (2), the generalized speeds, $u_{r}$, are

$$
u_{r}=\left(u_{1}, u_{2}, \ldots, u_{r}\right)=(\dot{x}, \dot{\phi}, \dot{\theta}) ; \quad r=n-m,
$$

The velocity, angular velocity and angular acceleration of point $C$ with respect to reference frame $F$ are 


$$
\begin{aligned}
& { }^{F} \hat{v}^{C}=u_{1} \mathbf{n}_{\mathbf{1}}, \\
& { }^{F} \hat{\omega}^{C}=u_{2} \mathbf{f}_{2}=u_{2} \mathbf{n}_{\mathbf{2}}, \\
& { }^{F} \hat{\alpha}^{C}=\dot{u}_{2} \mathbf{f}_{2}=\dot{u}_{2} \mathbf{n}_{\mathbf{2}},
\end{aligned}
$$

From (2), angular velocity of points $\mathrm{W}_{\tilde{\mathrm{P}} 1}$ and $\mathrm{W}_{\tilde{\mathrm{P}} 2}$ with respect to reference frame $F$ are

$$
\begin{aligned}
& { }^{F} \hat{\omega}^{W_{\tilde{P} 1}}=-u_{2} \frac{L}{R}\left(-S_{2} \mathbf{n}_{1}+C_{2} \mathbf{n}_{\mathbf{3}}\right)-u_{1} \frac{1}{R} \mathbf{n}_{3}, \\
& { }^{F} \hat{\omega}^{W_{\tilde{P} 2}}=u_{2} \frac{L}{R}\left(-S_{2} \mathbf{n}_{\mathbf{1}}+C_{2} \mathbf{n}_{\mathbf{3}}\right)-u_{1} \frac{1}{R} \mathbf{n}_{3},
\end{aligned}
$$

The angular velocity of robot main body and two wheels with respect to reference frame $F$ are

$$
\begin{aligned}
& { }^{F} \hat{\boldsymbol{\omega}}^{B}={ }^{F} \hat{\boldsymbol{\omega}}^{C}+{ }^{C} \hat{\omega}^{G}=u_{2} \mathbf{n}_{2}-u_{3} \mathbf{n}_{3}, \\
& { }^{F} \hat{\omega}^{W_{1}}={ }^{F} \hat{\omega}^{C}+{ }^{F} \hat{\omega}^{W_{\tilde{P} 1}}=-u_{1} \frac{1}{R} \mathbf{n}_{3}+u_{2}\left(\mathbf{n}_{\mathbf{2}}-\frac{L C_{2}}{R} \mathbf{n}_{3}+\frac{L S_{2}}{R} \mathbf{n}_{\mathbf{1}}\right) \\
& { }^{F} \hat{\omega}^{W_{2}}={ }^{F} \hat{\omega}^{C}+{ }^{F} \hat{\omega}^{W_{\tilde{P} 2}}=-u_{1} \frac{1}{R} \mathbf{n}_{3}+u_{2}\left(\mathbf{n}_{2}+\frac{L C_{2}}{R} \mathbf{n}_{3}-\frac{L S_{2}}{R} \mathbf{n}_{\mathbf{1}}\right)
\end{aligned}
$$

${ }^{C} \hat{\omega}^{G}$ is an angular velocity of point $G$ with respect to robot point $C$. Based on a rigid body motion solution in $3 \mathrm{D}$ space, the velocity of the COG of the main body and the centre of two wheels with respect to reference frame $F$ can be formulated as

$$
{ }^{F} \hat{v}^{P i}={ }^{F} \hat{v}^{C}+{ }^{F} \hat{\omega}^{C} \times \hat{r}^{P i}+{ }^{C} \hat{v}^{P i} ; \quad\left(P_{i}=G, W_{C 1}, W_{C 2}\right),
$$

$\hat{r}^{P i}$ and ${ }^{C} \hat{v}^{P i}$ are the displacement and velocity of point $P_{i}$ with respect to point $\mathrm{C}$. The centre of wheels are fixed with respect to point $C$, i.e ${ }^{C} \hat{v}^{W_{C i}}=0$, Thus

$$
\begin{aligned}
& { }^{F} \hat{v}^{G}={ }^{F} \hat{v}^{C}+{ }^{F} \hat{\omega}^{C} \times{ }^{C} \hat{d}^{G}+{ }^{C} \hat{\omega}^{G} \times{ }^{C} \hat{d}^{G}=u_{1} \mathbf{n}_{\mathbf{1}}-u_{2} d S_{3} \mathbf{n}_{\mathbf{3}}+u_{3}\left(d C_{3} \mathbf{n}_{\mathbf{1}}-d S_{3} \mathbf{n}_{\mathbf{2}}\right), \\
& { }^{F} \hat{v}^{W_{C 1}}={ }^{F} \hat{v}^{C}+{ }^{F} \hat{\omega}^{C} \times{ }^{C} \hat{L}^{W_{C 1}}=u_{1} \mathbf{n}_{\mathbf{1}}+u_{2} L \mathbf{n}_{\mathbf{1}}, \\
& { }^{F} \hat{v}^{W_{C 2}}={ }^{F} \hat{v}^{C}+{ }^{F} \hat{\omega}^{C} \times{ }^{C} \hat{L}^{W_{C 2}}=u_{1} \mathbf{n}_{\mathbf{1}}-u_{2} L \mathbf{n}_{\mathbf{1}},
\end{aligned}
$$

${ }^{C} \hat{d}^{G}$ and ${ }^{C} \hat{L}^{W_{C i}}$ are displacements of the COG of main body and the centre of wheels with respect to point $\mathrm{C}$. The angular acceleration of the main body and the wheels are

$$
\begin{aligned}
& { }^{F} \hat{\alpha}^{B}=\frac{d^{F} \hat{\omega}^{B}}{d t}=\dot{u}_{2} \mathbf{n}_{2}-\dot{u}_{3} \mathbf{n}_{3}, \\
& { }^{F} \hat{\alpha}^{W_{1}}=\frac{d^{F} \hat{\omega}^{W_{1}}}{d t}+{ }^{F} \hat{\omega}^{C} \times{ }^{F} \hat{\omega}^{W_{\tilde{P} 1}}=\left(\frac{\dot{u}_{2} L S_{2}-u_{1} u_{2}}{R}\right) \mathbf{n}_{1}+\dot{u}_{2} \mathbf{n}_{2}-\frac{\dot{u}_{1}+\dot{u}_{2} L C_{2}}{R} \mathbf{n}_{3}, \\
& { }^{F} \hat{\alpha}^{W_{2}}=\frac{d^{F} \hat{\omega}^{W_{2}}}{d t}+{ }^{F} \hat{\omega}^{C} \times{ }^{F} \hat{\omega}^{W_{\tilde{P} 2}}=\left(\frac{-\dot{u}_{2} L S_{2}-u_{1} u_{2}}{R}\right) \mathbf{n}_{1}+\dot{u}_{2} \mathbf{n}_{2}-\frac{\dot{u}_{1}-\dot{u}_{2} L C_{2}}{R} \mathbf{n}_{3},
\end{aligned}
$$


From (7) the acceleration of the COG of main body and centre of two wheels with respect to reference frame $F$ can be formulated as

$$
{ }^{F} \hat{a}^{P i}={ }^{F} \hat{a}^{C}+{ }^{F} \hat{\alpha}^{C} \times \hat{r}^{P i}+{ }^{F} \hat{\omega}^{C} \times\left({ }^{F} \hat{\omega}^{C} \times \hat{r}^{P i}\right)+{ }^{F} \hat{\omega}^{C} \times{ }^{C} \hat{v}^{P i}+{ }^{C} \hat{a}^{P i} ;\left(P_{i}=G, W_{C 1}, W_{C 2}\right),
$$

${ }^{F} \hat{a}^{C}$ is an acceleration of point $\mathrm{C}$ with respect to frame $F$ and ${ }^{C} \hat{a}^{P i}=d^{C} \hat{v}^{P i} / d t$ is an acceleration of point $\mathrm{P}_{\mathrm{i}}$ with respect to point $\mathrm{C}$. Based on aforementioned fact, ${ }^{C} \hat{a}^{W_{C i}}=0$, thus

$$
\begin{aligned}
& { }^{F} \hat{a}^{G}=\frac{d^{F} \hat{v}^{C}}{d t}+{ }^{F} \hat{\alpha}^{C} \times{ }^{C} \hat{d}^{G}+{ }^{F} \hat{\omega}^{C} \times\left({ }^{F} \hat{\omega}^{C} \times{ }^{C} \hat{d}^{G}\right)+{ }^{F} \hat{\omega}^{C} \times{ }^{C} \hat{v}^{G}+{ }^{C} \hat{a}^{G}, \\
& =\left(\dot{u}_{1}+\dot{u}_{3} d C_{3}-u_{2}^{2} d S_{3}-u_{3}^{2} d S_{3}\right) \mathbf{n}_{1}-\left(\dot{u}_{3} d S_{3}+u_{3}^{2} d C_{3}\right) \mathbf{n}_{2}-\left(\dot{u}_{2} d S_{3}+u_{2} u_{3} d C_{3}\right) \mathbf{n}_{3}, \\
& { }^{F} \hat{a}^{W_{C 1}}=\frac{d^{F} \hat{v}^{C}}{d t}+{ }^{F} \hat{\alpha}^{C} \times{ }^{C} \hat{L}^{W_{C 1}}+{ }^{F} \hat{\omega}^{C} \times\left({ }^{F} \hat{\omega}^{C} \times{ }^{C} \hat{L}^{W_{C 1}}\right)=\left(\dot{u}_{1}+\dot{u}_{2} L\right) \mathbf{n}_{1}-u_{2}^{2} L \mathbf{n}_{3}, \\
& { }^{F} \hat{a}^{W_{C 2}}=\frac{d^{F} \hat{v}^{C}}{d t}+{ }^{F} \hat{\alpha}^{C} \times C{ }^{C} W^{C 2}+{ }^{F} \hat{\omega}^{C} \times\left({ }^{F} \hat{\omega}^{C} \times{ }^{C} \hat{L}^{W_{C 2}}\right)=\left(\dot{u}_{1}-\dot{u}_{2} L\right) \mathbf{n}_{1}+u_{2}^{2} L \mathbf{n}_{3},
\end{aligned}
$$

Partial velocity and partial angular velocity of the main body and the wheels can be obtained by differentiating (4), (6) and (8) with $u_{r}$ as shown below.

$$
\begin{aligned}
& { }_{\tilde{v}_{r}}^{P i}=\frac{\partial^{F} \hat{v}^{P i}}{\partial u_{r}} ; \quad(r=1,2,3),\left(P_{i}=G, C, W_{C 1}, W_{C 2}\right), \\
& F \widetilde{\omega}_{r}^{B i}=\frac{\partial^{F} \hat{\omega}^{B i}}{\partial u_{r}} ; \quad(r=1,2,3),\left(B_{i}=B, A, W_{1}, W_{2}\right),
\end{aligned}
$$

\subsection{Dynamic of WIP}

WIP lies under differential drive type of mobile robot. Thus generated torque, $\tau_{\mathrm{C}}$, from the motors to the robot can be represented as

$$
\tau_{C}=\tau_{\text {pitch }}+\tau_{\text {yaw }}
$$

The yaw torque, $\tau_{\text {yaw }}$, and pitch torque, $\tau_{\text {pitch }}$, are the torques that cause WIP steers by,$\phi$, in $\mathbf{f}_{\mathbf{2}}$ directional and move with velocity $v_{C}$ in $\mathbf{n}_{\mathbf{1}}$ directional as shown in Fig. 1(b). However, pitch torque exerts to robot main body in the form of force. Therefore at every instant, this robot experiences both forward force and steering torque. Based on Fig. 1(b) and the assumptions, the active forces are

$$
\begin{aligned}
& \hat{R}_{C}=\gamma_{1} \mathbf{n}_{\mathbf{1}}+\gamma_{2} \mathbf{n}_{\mathbf{2}}+\gamma_{3} \mathbf{n}_{\mathbf{3}}, \\
& \hat{T}_{C}=\delta_{1} \mathbf{n}_{\mathbf{1}}+\delta_{2} \mathbf{n}_{\mathbf{2}}+\delta_{3} \mathbf{n}_{\mathbf{3}}, \\
& \hat{R}_{w_{1}}=-m_{W} g \mathbf{n}_{\mathbf{2}}, \\
& \hat{R}_{w_{2}}=-m_{W} g \mathbf{n}_{\mathbf{2}}, \\
& \hat{R}_{B}=-m_{B} g \mathbf{n}_{\mathbf{2}},
\end{aligned}
$$


Where

$$
\begin{aligned}
& \gamma_{i}=\left\{\begin{array}{cc}
F_{w r}+F_{w l}=\frac{\tau_{w r}+\tau_{w l}}{R} ; & i=1, \\
0 & \text { otherwise, }
\end{array}\right. \\
& \delta_{i}=\left\{\begin{array}{cc}
\left(F_{w r}-F_{w l}\right) L C_{2}=\left(\tau_{w r}-\tau_{w l}\right) \frac{L}{R} C_{2} ; & i=2, \\
0 & \text { otherwise, }
\end{array}\right.
\end{aligned}
$$

$\hat{R}_{C}$ and $\hat{T}_{C}$ are the force and torque exerted on the main body by wheel, $\hat{R}_{w_{1}}, \hat{R}_{w_{2}}$ and $\hat{R}_{B}$ are forces exerted on the wheel and body by the gravity. $F_{w r}$ and $F_{w l}$ are translated forces from the wheels torque. The inertia force and inertia torque of the main body and the wheels are

$$
\begin{aligned}
& \hat{R}_{P i}^{*}=-m_{P i}{ }^{F} \hat{a}^{P i} ;\left(P_{i}=G, W_{C 1}, W_{C 2}\right), \\
& \hat{T}_{B i}{ }^{*}=-{ }^{F} \hat{\alpha}^{B i} \cdot \hat{\hat{I}}_{B i}-{ }^{F} \hat{\omega}^{B i} \times \hat{\hat{I}}_{B i}{ }^{F} \hat{\omega}^{B i} ;\left(B_{i}=B, W_{1}, W_{2}\right),
\end{aligned}
$$

Where $m_{G}=m_{B}, m_{W_{C i}}=m_{W}, \hat{\hat{I}}_{B i}=I_{b 2} \mathbf{n}_{\mathbf{2}} \mathbf{n}_{\mathbf{2}}+I_{b 3} \mathbf{n}_{\mathbf{3}} \mathbf{n}_{\mathbf{3}}$ and $\hat{\hat{I}}_{W_{i}}=I_{W_{2}} \mathbf{n}_{\mathbf{2}} \mathbf{n}_{\mathbf{2}}+I_{W_{3}} \mathbf{n}_{\mathbf{3}} \mathbf{n}_{\mathbf{3}}$. The inertia of the wheels is considered as $I_{W_{2}}=m_{W} R^{2} / 4+m_{W} L^{2}$ and $I_{W_{3}}=m_{W} R^{2} / 4$. The generalized active force and inertia force are given as

$$
\begin{aligned}
& F_{r}=\sum_{i=1}^{j} F \tilde{v}_{r}^{P i} \cdot \hat{R}_{P i}+\sum_{i=1}^{k} F \widetilde{\omega}_{r}^{B i} \cdot \hat{T}_{B i} ; \quad(r=1,2,3),\left(P_{i}=G, C, W_{C 1}, W_{C 2}\right),\left(B_{i}=C\right), \\
& F_{r}^{*}=\sum_{i=1}^{j} F \widetilde{v}_{r}^{P i} \cdot \hat{R}_{P i}^{*}+\sum_{i=1}^{k}{ }^{F} \widetilde{\omega}_{r}^{B i} \cdot \hat{T}_{B i}^{*} ;(r=1,2,3),\left(P_{i}=G, W_{C 1}, W_{C 2}\right),\left(B_{i}=B, W_{1}, W_{2}\right),
\end{aligned}
$$

Where $\mathrm{j}$ is the number of points subjected to applied forces and $\mathrm{k}$ is the number of rigid bodies subjected to applied torques. Three motion equations of WIP (19) are obtained by equating the dynamic equation of motion (18),

$$
\begin{aligned}
& F_{r}+F_{r}^{*}=0, \\
& \left(m_{b}+2 m_{w}+2 \frac{I_{w 3}}{R^{2}}\right) \ddot{x}+m_{b} d C_{3} \ddot{\theta}=\frac{\tau_{w r}+\tau_{w l}}{R}+\left(\dot{\phi}^{2}+\dot{\theta}^{2}\right) m_{b} d S_{3}, \\
& \left(m_{b}\left(d S_{3}\right)^{2}+2 m_{w} L^{2}+I_{b 2}+2 I_{w 2}+2 \frac{\left(L C_{2}\right)^{2}}{R^{2}} I_{w 3}\right) \ddot{\phi}=\left(\tau_{w r}-\tau_{w l}\right) \frac{L C_{2}}{R}-\dot{\theta} \dot{\phi} m_{b} d^{2} S_{3} C_{3}, \\
& m_{b} d C_{3} \ddot{x}+\left(m_{b} d^{2}+I_{b 3}\right) \ddot{\theta}=m_{b} d g S_{3}+\dot{\phi}^{2} m_{b} d^{2} S_{3} C_{3},
\end{aligned}
$$


Then state space representation of the WIP (20) is solved using Cramer rule. The matrix variables are listed as in the appendix.

$$
\begin{aligned}
& \dot{\mathbf{x}}(t)=\mathbf{A}(\mathbf{x}, t) \mathbf{x}(t)+\mathbf{B}(\mathbf{x}, t) \mathbf{u}(t)+f(\mathbf{x}, t) \\
& {\left[\begin{array}{c}
\dot{x} \\
\ddot{x} \\
\dot{\theta} \\
\ddot{\theta} \\
\dot{\phi} \\
\ddot{\phi}
\end{array}\right]=\left[\begin{array}{cccccc}
0 & 1 & 0 & 0 & 0 & 0 \\
0 & 0 & 0 & A_{24} & 0 & A_{26} \\
0 & 0 & 0 & 1 & 0 & 0 \\
0 & 0 & 0 & A_{44} & 0 & A_{46} \\
0 & 0 & 0 & 0 & 0 & 1 \\
0 & 0 & 0 & 0 & 0 & A_{66}
\end{array}\right]\left[\begin{array}{c}
x \\
\dot{x} \\
\theta \\
\dot{\theta} \\
\phi \\
\dot{\phi}
\end{array}\right]+\left[\begin{array}{cc}
0 & 0 \\
B_{21} & B_{22} \\
0 & 0 \\
B_{41} & B_{42} \\
0 & 0 \\
B_{61} & B_{62}
\end{array}\right]\left[\begin{array}{c}
\tau_{w r} \\
\tau_{w l}
\end{array}\right]+\left[\begin{array}{c}
0 \\
F_{2} \\
0 \\
F_{4} \\
0 \\
0
\end{array}\right],}
\end{aligned}
$$

\section{Partial State-PID controller}

Notation: Throughout this section, i index indicates the balancing and heading subsystems by equalized it to $\theta$ and $\phi$.

\subsection{Linear WIP model}

To design a linear controller, the nonlinear WIP model is linearized by setting tilt angle, $\theta \approx 0$, and heading angle, $\phi \approx 0$. The linear WIP system can be represented into two subsystems, first is "balancing" subsystem which is regarding the rotation and motion about $\mathbf{n}_{\mathbf{3}}$ and $\mathbf{n}_{1}$ directional and second is "heading" subsystem which is describing the rotation about $\mathbf{n}_{\mathbf{2}}$ directional. These subsystems can be formulated as

$$
\begin{aligned}
\dot{\mathbf{x}}_{i}(t) & =\mathbf{A}_{i} \mathbf{x}_{i}(t)+\mathbf{B}_{i} \mathbf{u}_{i}(t) ; \quad(i=\theta, \phi), \\
\mathbf{y}_{i}(t) & =\mathbf{C}_{i} \mathbf{x}_{i}(t)
\end{aligned}
$$

Here $\mathbf{A}_{i}, \mathbf{B}_{i}$ and $\mathbf{C}_{i}$ are state constant system matrix, system input matrix and system output matrix with appropriate dimension. $\mathbf{x}_{i} \in \mathbb{R}^{n_{i}}$ and $u_{i} \in \mathbb{R}$ are defined as state variable and control input with $n_{i}$ number of state variables. The state vector of balancing and heading subsystems are $\mathbf{x}_{\theta}=(x, \dot{x}, \theta, \dot{\theta})$ and $\mathbf{x}_{\phi}=(\phi, \dot{\phi})$. The characteristic polynomial of both subsystems given as

$$
\operatorname{det}\left(s \mathbf{I}-\mathbf{A}_{i}\right)=a_{i 0} s^{n_{i}}+a_{i 1} s^{n_{i}-1}+\cdots+a_{i\left(n_{i}-1\right)} s+a_{i n_{i}},
$$

Where $\mathbf{a}_{i}=\left[\begin{array}{llll}a_{i 0} & a_{i 1} & \ldots & a_{i n_{i}}\end{array}\right]$ is a characteristic vector with coefficients $a_{i 0}=1, a_{i n_{i}}=\operatorname{det}\left(-\mathbf{A}_{i}\right)$ and $a_{i 1}=-\operatorname{trace}\left(-\mathbf{A}_{i}\right)$. Both balancing and heading subsystems are

$$
\begin{aligned}
& {\left[\begin{array}{c}
\dot{x} \\
\ddot{x} \\
\dot{\theta} \\
\ddot{\theta}
\end{array}\right]=\left[\begin{array}{cccc}
0 & 1 & 0 & 0 \\
0 & 0 & A_{23 L} & 0 \\
0 & 0 & 0 & 1 \\
0 & 0 & A_{43 L} & 0
\end{array}\right]\left[\begin{array}{c}
x \\
\dot{x} \\
\theta \\
\dot{\theta}
\end{array}\right]+\left[\begin{array}{c}
0 \\
B_{21 L} \\
0 \\
B_{41 L}
\end{array}\right] u_{\theta},} \\
& {\left[\begin{array}{c}
\dot{\phi} \\
\ddot{\phi}
\end{array}\right]=\left[\begin{array}{ll}
0 & 1 \\
0 & 0
\end{array}\right]\left[\begin{array}{c}
\phi \\
\dot{\phi}
\end{array}\right]+\left[\begin{array}{c}
0 \\
B_{61 L}
\end{array}\right] u_{\phi},}
\end{aligned}
$$


Equation (23) showed $n_{\theta}=4$ for balancing subsystem and $n_{\phi}=2$ for heading subsystem. The matrix variables are listed as in the appendix. Using decoupling technique, the relationship of control input and $u_{\phi}$ to the wheel torques are

$$
\begin{aligned}
& \tau_{w r}=0.5 u_{\theta}+0.5 u_{\phi}, \\
& \tau_{w l}=0.5 u_{\theta}-0.5 u_{\phi},
\end{aligned}
$$

\subsection{Augment subsystem with PSPID}

Fig. 2 shows closed loop WIP system. In such $n_{i}$ order system, input matrix, $\mathbf{r}_{i}$, and proposed PSPID controller are

$$
\begin{aligned}
& \mathbf{r}_{i}^{T}=\left[\begin{array}{llllll}
r_{i 1} & 0 & \ldots & r_{i(n-1)} & 0
\end{array}\right] ; \quad(i=\theta, \phi), \\
& \mathbf{K}_{\mathbf{P I D i}}=\left[\begin{array}{l}
\mathbf{K}_{\mathbf{P} i} \\
\mathbf{K}_{\mathbf{I} i} \\
\mathbf{K}_{\mathbf{D} i}
\end{array}\right]=\left[\begin{array}{llllll}
K_{P i 1} & 0 & K_{P i 3} & \cdots & K_{P i(n-1)} & 0 \\
K_{l i 1} & 0 & K_{l i 3} & \cdots & K_{I i(n-1)} & 0 \\
K_{D i 1} & 0 & K_{D i 3} & \cdots & K_{D i(n-1)} & 0
\end{array}\right],
\end{aligned}
$$

$r_{i 1}, \ldots, r_{i\left(n_{i}-1\right)}$ are the input variables, $\mathbf{K}_{\mathbf{P} i}, \mathbf{K}_{\mathbf{I} i}$, and $\mathbf{K}_{\mathbf{D} i}$ are proportional, integral and derivative gain vector of PSPID controller. Based on (23) and (25), the PID controllers for the balancing and heading subsystems are $\quad \mathbf{K}_{\mathbf{P I D} \theta 1}=\left[\begin{array}{llll}K_{P \theta 1} & K_{1 \theta 1} & K_{D \theta 1}\end{array}\right]^{T}, \quad \mathbf{K}_{\mathbf{P I D} \theta 3}=\left[\begin{array}{lll}K_{P \theta 3} & K_{1 \theta 3} & K_{D \theta 3}\end{array}\right]^{T} \quad$ and $\mathbf{K}_{\mathbf{P I D} \phi 1}=\left[\begin{array}{lll}K_{P \phi 1} & K_{I \phi 1} & K_{D \phi 1}\end{array}\right]^{T}$. The control input $u_{i}$ is

$$
u_{i}=\mathbf{K}_{\mathbf{P}_{i}}\left(\mathbf{r}_{i}-\mathbf{y}_{i}\right)+\mathbf{K}_{\mathbf{I}_{i}} \int\left(\mathbf{r}_{i}-\mathbf{y}_{i}\right) d t+\mathbf{K}_{\mathbf{D}_{i}} \frac{d}{d t}\left(\mathbf{r}_{i}-\mathbf{y}_{i}\right)
$$

The output matrix is $\mathbf{y}_{i}(t)=\mathbf{C}_{i} \mathbf{x}_{i}(t)=\mathbf{x}_{i n_{o}}(t)$ where $\mathbf{x}_{i n_{o}}=\left[\begin{array}{llllll}x_{i 1} & 0 & \ldots & x_{i\left(n_{i}-1\right)} & 0\end{array}\right]^{T}$. For simplicity, set all input variable to zero $\left(\mathbf{r}_{i}=\mathbf{0}_{n_{i}}\right)$, hence the control input is

$$
u_{i}=-\mathbf{K}_{\mathbf{P D} i n} \mathbf{x}_{i}-\mathbf{K}_{\mathbf{I} i n} \int \mathbf{y}_{i} d t
$$

$=\mathrm{y}$ is defined, hence (27) will be

$$
u_{i}=-\mathbf{K}_{\mathbf{p} i n} \mathbf{x}_{i}-\mathbf{K}_{\mathbf{I} i n} \mathbf{s}_{i},
$$

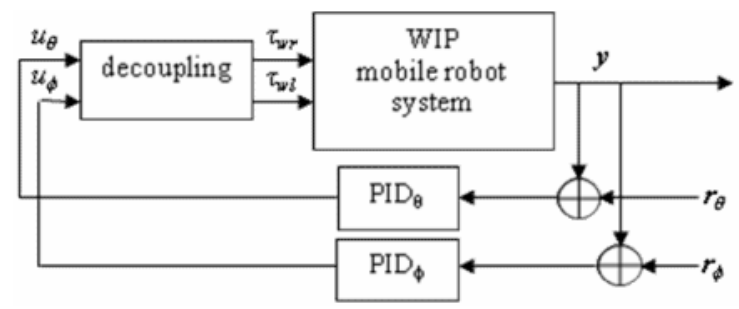

Figure 2. Closed loop WIP structure with PSPID.

The second term in (28) can be simplified to a scalar by a following formula

$$
\begin{aligned}
& K_{I i}^{\prime} \mathrm{s}_{i}^{\prime}=K_{I i}^{\prime}\left(\beta_{i 1} \mathrm{~s}_{i 1}+\beta_{i 3} \mathrm{~s}_{i 3}+\cdots+\beta_{i\left(n_{i}-1\right)} \mathrm{s}_{i\left(n_{i}-1\right)}\right), \\
& =K_{I i 1} \varsigma_{i 1}+K_{I i 3} \varsigma_{i 3}+\cdots+K_{I i\left(n_{i}-1\right)} \varsigma_{i\left(n_{i}-1\right)},
\end{aligned}
$$


$\beta_{i n_{i}}$ is a coefficient with arbitrary selected value. Thus input signal $u_{i}$ is

$$
\begin{aligned}
u_{i} & =-\overline{\mathbf{K}}_{i} \overline{\mathbf{x}}_{i} ; \\
\overline{\mathbf{K}}_{i} & =\left[\begin{array}{lllll}
K_{P_{i} 1} & K_{D_{i} 1} & \cdots & K_{D_{i}\left(n_{i}-1\right)} & K_{I_{i}}^{\prime}
\end{array}\right], \\
\overline{\mathbf{x}}_{i}^{T} & =\left[\begin{array}{lll}
x_{i 1}(t), \cdots & x_{i n_{i}}(t), s_{i}^{\prime}(t)
\end{array}\right]
\end{aligned}
$$

It is obvious in (29) $s_{i}{ }^{\prime}=\boldsymbol{\beta}_{i} s_{i}$, thus

$$
\begin{aligned}
\dot{s}_{i} & =\boldsymbol{\beta}_{i} \dot{\mathbf{s}}_{i}=\boldsymbol{\beta}_{i} \mathbf{C}_{i} \mathbf{x}_{i}=\mathbf{C}_{\boldsymbol{\beta}_{i} \mathbf{x}_{i} ;} \\
\mathbf{C}_{\boldsymbol{\beta}_{i}} & =\left[\begin{array}{lllll}
\beta_{i 1} & 0 & \cdots & \beta_{i\left(n_{i}-1\right)} & 0
\end{array}\right]
\end{aligned}
$$

Based on above formulation, equivalent $n_{i}+1$ order subsystem dynamic can be formulated from the combination of the original state (23) and (31). The $n_{i}+1$ state vector is written as

$$
\begin{aligned}
& \dot{\overline{\mathbf{x}}}_{i}=\overline{\mathbf{A}}_{i} \overline{\mathbf{x}}_{i}+\overline{\mathbf{B}}_{i} u_{i}, \\
& {\left[\begin{array}{c}
\dot{\mathbf{x}}_{i}(t) \\
\dot{s}_{i}(t)
\end{array}\right]=\left[\begin{array}{cc}
\mathbf{A}_{i} & 0 \\
\mathbf{C}_{\boldsymbol{\beta}_{i}} & 0
\end{array}\right]\left[\begin{array}{c}
\mathbf{x}_{i}(t) \\
s_{i}(t)
\end{array}\right]+\left[\begin{array}{c}
\mathbf{B}_{i} \\
0
\end{array}\right] u_{i}(t),}
\end{aligned}
$$

Here $\beta_{i n_{i}}$ is set to one. The output matrix $\overline{\mathbf{C}}_{i}$ is set to

$$
\overline{\mathbf{C}}_{i}=\left[\begin{array}{cc}
\mathbf{C}_{i} & 0 \\
0 & 1
\end{array}\right]
$$

The rank of controllability matrix $\quad\left[\begin{array}{llllll}\overline{\mathbf{B}}_{i} & \overline{\mathbf{A}}_{i} \overline{\mathbf{B}}_{i} & \overline{\mathbf{A}}_{i}^{2} \overline{\mathbf{B}}_{i} & \cdots & \overline{\mathbf{A}}_{i}^{n} \overline{\mathbf{B}}_{i}\end{array}\right]$ of system (32) is $n_{i}+1$. This means the linear system (32) is completely controllable.

\subsection{Optimal PSPID}

To apply QOR tuning method, Frobenius Canonical form of system (32) is considered by following transformation.

$$
\mathbf{z}_{i}=\mathbf{T}_{i} \overline{\mathbf{x}}_{i},
$$

Where $\mathbf{z}_{i}\left(m_{i} \times 1\right)$ and $\mathbf{T}_{i}\left(m_{i} \times m_{i}\right)$ are transformation state variable vector and transformation matrix. Index $m_{i}=n_{i}+1$, and $\mathbf{T}_{i}$ matrix define as

$$
\mathbf{T}_{i}=\left[\begin{array}{c}
\mathbf{q}_{i 1} \\
\mathbf{q}_{i 1} A_{i} \\
\vdots \\
\mathbf{q}_{i 1} A_{i}^{n_{i}-1}
\end{array}\right] ; \quad \mathbf{q}_{i 1}=\mathbf{e}_{i n_{i}}^{T} \mathbf{W}_{c_{i}}^{-1},
$$

$\mathbf{q}_{i} \mathbf{1} \quad$ is $1 \times m_{i}$ vector, $\quad \mathbf{W}_{c_{i}}=\left[\begin{array}{lllll}\overline{\mathbf{B}}_{i} & \overline{\mathbf{A}}_{i} \overline{\mathbf{B}}_{i} & \cdots & \overline{\mathbf{A}}_{i}^{m_{i}} \overline{\mathbf{B}}_{i}\end{array}\right]$ and unit vector $\mathbf{e}_{i n_{i}}=\left[\begin{array}{llll}0 & 0 & \cdots & 1\end{array}\right]$, the Frobenius canonical form of WIP subsystems can be express as

$$
\dot{\mathbf{z}}_{i}=\mathbf{A}_{\mathbf{C} i} \mathbf{z}_{i}+\mathbf{B}_{\mathbf{C} i} u_{i},
$$


Where

$$
\begin{aligned}
& \mathbf{A}_{\mathbf{c} i}=\mathbf{T}_{i} \overline{\mathbf{A}}_{i} \mathbf{T}_{i}^{-1}=\left[\begin{array}{ccccc}
0 & 1 & 0 & \cdots & 0 \\
0 & 0 & 1 & \cdots & 0 \\
\vdots & \vdots & \ddots & \ddots & \vdots \\
0 & 0 & 0 & \cdots & 1 \\
-\alpha_{i m_{i}} & -\alpha_{i\left(m_{i}-1\right)} & -\alpha_{i\left(m_{i}-2\right)} & \cdots & -\alpha_{i 1}
\end{array}\right], \\
& \mathbf{B}_{\mathbf{c} i}=\mathbf{T}_{i} \overline{\mathbf{B}}_{i}=\left[\begin{array}{c}
0 \\
0 \\
\vdots \\
1
\end{array}\right],
\end{aligned}
$$

The input signal is

$$
\begin{aligned}
& u_{i}=-\mathbf{K}_{\mathbf{C} i} \mathbf{z}_{i} ; \\
& \overline{\mathbf{K}}_{i}=\left[\begin{array}{lllll}
K_{C i 1} & K_{C i 2} & \cdots & K_{C i\left(m_{i}-1\right)} & K_{C i m_{i}}
\end{array}\right] \\
& \mathbf{z}_{i}^{T}=\left[z_{i 1}(t), z_{i 2}(t) \cdots z_{i m_{i}}(t)\right]
\end{aligned}
$$

And also feedback gain

$$
\mathbf{K}_{\mathbf{C} i}=\overline{\mathbf{K}}_{i} \mathbf{T}_{i}^{-1}
$$

The desired $m$ order characteristic equation of matrix $\mathbf{A}_{\mathbf{C} i}-\mathbf{B}_{\mathbf{C} i} \mathbf{K}_{\mathbf{C} i}$ is

$$
\operatorname{det}\left(s I-A_{i}\right)=s^{m_{i}}+\left(\alpha_{i 1}+K_{c i m_{i}}\right) s^{m_{i}-1}+\cdots+\left(\alpha_{i\left(m_{i}-1\right)}+K_{c i 2}\right) s+\left(\alpha_{i m_{i}}+K_{c i 1}\right)
$$

As shown, system (32) is completely controllable, then $\mathbf{K}_{\mathbf{C} i}$ can be tuned by QOR method. The QOR problem is a quadratic performance index function for the integral function. The index is in the form of quadratic performance of continuous-time linear time-varying function.

$$
I=\frac{1}{2} \mathbf{z}_{i}^{T}\left(t_{f}\right) \mathbf{P}_{\mathbf{f i}} \mathbf{z}_{i}\left(t_{f}\right)+\frac{1}{2} \int_{0}^{t_{f}}\left(\mathbf{z}_{i}^{T}(t) \mathbf{Q}_{i} \mathbf{z}_{i}(t)+u_{i}^{T}(t) \mathbf{R}_{i} u_{i}(t)\right) d t
$$

Where the final time, $t_{f}$, is fix. $\mathbf{P}_{\mathbf{f}_{i}}, \mathbf{Q}_{i}$, are chosen to be symmetry positive semidefinite, and $\mathbf{R}_{i}$ to be symmetry positive definite. The QOR optimal control problem is to drive the state vector $\mathbf{z}_{i}(t)$ to the origin from any nonzero initial values of states. The problem objective is to find $u_{i}(t)$; $0 \leq t \leq t_{f}$, such that the objective function (41) is minimized. Choosing the appropriate weighting matrix $\mathbf{Q}_{i}, \mathbf{R}_{i}$, and therefore get the optimal control rate with optimal control theory. For an infinite time $t_{f}, \mathbf{P}_{\mathrm{f} i}$ must satisfy following reduced Riccati equation.

$$
\mathbf{A}_{\mathrm{ci} i}^{T} \mathbf{P}_{\mathrm{f} i}+\mathbf{P}_{\mathbf{f}} \mathbf{A}_{\mathrm{c} i}-\mathbf{P}_{\mathrm{fi}} \mathbf{B}_{\mathrm{c} i} \mathbf{R}_{i}{ }^{-1} \mathbf{B}_{\mathrm{c} i}^{T} \mathbf{P}_{\mathrm{f} i}+\mathbf{Q}_{i}=0
$$

With predetermined weighted matrix, optimal control is

$$
u_{i}(t)=-\mathbf{R}_{i}^{-1} \mathbf{B}_{\mathbf{c} i}^{T} \mathbf{P}_{\mathbf{f} i} \mathbf{z}_{i}\left(t_{f}\right)=-\mathbf{K}_{\mathbf{C} i} \mathbf{z}_{i}\left(t_{f}\right)
$$


The control law makes the linear feedback system stable, then by adopting Lyapunov second law, the nonlinear feedback system is stable. Weighting matrix $\mathbf{Q}_{i}$ and $\mathbf{R}_{i}$ are

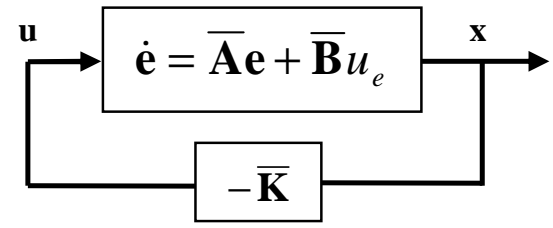

Figure 3. Equivalent $n_{i}+1$ order error vector of system (2) with PID controller

$$
Q=\left[\begin{array}{cccc}
q_{11} & 0 & \cdots & 0 \\
0 & q_{22} & \ddots & \vdots \\
\vdots & \ddots & \ddots & 0 \\
0 & \cdots & 0 & q_{m_{i} m_{i}}
\end{array}\right] ; R=\left[r_{11}\right]
$$

Finally, based on (39) and (30), PSPID controller is directly tuned for subsystems (23). The formulation makes the proposed controller has both of PID controller and QOR advantages.

Following steps can be applied to tune PID controller using QOR technique:

Step 1. Construct $m_{i}$ order system (32) from the combination of the original system (23) and (31). Step 2. Transform this $m_{i}$ order system to a Frobenius Canonical form based on transformation formula (34).

Step 3. Tune $\mathbf{K}_{\mathrm{c} i}$ gain by appropriately tuning $\mathbf{Q}_{i}$ and $\mathbf{R}_{i}$ (44) matrices.

Step 4. Compute $\overline{\mathbf{K}}_{i}$ in (30) from $\mathbf{K}_{\mathrm{c} i}$ by transformation formulation (14).

Step 5. Lastly Based on (30), set PID gains (25) accordingly.

\section{RESULT AND DISCUSSION}

\subsection{Model analysis}

In QOR tuning scheme, $\quad \mathbf{Q}_{\theta}=\operatorname{diag}(83.112,2.583,5.2287,3.4656), \quad \mathbf{Q}_{\phi}=\operatorname{diag}(0.01,1) \quad$ and $\mathbf{R}_{\theta}=\mathbf{R}_{\phi}=1 \times 10^{-3}$ were set for LQR controllers. For PSPID controller, the matrices $\mathbf{Q} s$ are $\mathbf{Q}_{\theta}=\operatorname{diag}(83.112,2.583,5.2287,3.4656,0.01), \quad \mathbf{Q}_{\phi}=\operatorname{diag}(0.01,1,0.01)$ and $\quad \mathbf{R}_{\theta}=\mathbf{R}_{\phi}=1 \times 10^{-3} . \quad$ Thus the following LQR and PSPID controller are obtained:

$$
\begin{aligned}
& \mathbf{K}_{\theta}=-[91.2,106.8,493.4,129] ; \mathbf{K}_{\phi}=-[493.4,12], \\
& \mathbf{K}_{\mathbf{P I D} \theta 1}=\left[\begin{array}{c}
-27.1 \\
-18.3 \\
-18.9
\end{array}\right] ; \mathbf{K}_{\mathbf{P I D} \theta 3}=\left[\begin{array}{c}
-106.0 \\
-18.3 \\
-21.5
\end{array}\right], \\
& \mathbf{K}_{\mathbf{P I D} \phi 1}=\left[\begin{array}{c}
11.8 \\
1.1 \\
3.1
\end{array}\right],
\end{aligned}
$$




\subsubsection{Open loop stability}

The stability of WIP depends on the balancing of its main body. To investigate this behaviour, the pole analysis of the balancing submatrix, $\mathbf{A}_{\theta}^{\prime}$, from constant matrix $\mathbf{A}_{\theta}$ was conducted. The poles are obtained by solving following equation

$$
\operatorname{det}\left[s \mathbf{I}_{n_{\theta}}-\mathbf{A}_{\theta}^{\prime}\right]=\operatorname{det}\left[\begin{array}{cc}
s & -1 \\
-A_{43 L} & s
\end{array}\right]=0,
$$

Thus the poles are

$$
\begin{aligned}
& s_{\theta 1}=\sqrt{\frac{\left(m_{B}+3 m_{W}\right) m_{b} d g}{\left(m_{B}+3 m_{W}\right)\left(m_{b} d^{2}+I_{B 3}\right)-\left(m_{B} d\right)^{2}}}, \\
& s_{\theta 2}=-\sqrt{\frac{\left(m_{B}+3 m_{W}\right) m_{b} d g}{\left(m_{B}+3 m_{W}\right)\left(m_{b} d^{2}+I_{B 3}\right)-\left(m_{B} d\right)^{2}}},
\end{aligned}
$$

This showed one of its pole was positive. The fact shows, if the WIP not accelerates (ie. $u_{\theta}=0$ ), the robot body will fall. Another fact is the mass of robot body, the mass of the wheel and the COG of the main body will determine the magnitude of this pole.

\subsubsection{Effect of main body COG on tilt angle, $\theta$, control}

Firstly, robot body was assumed as a point of mass, $I_{b 3}=m_{b} d^{2}$. Therefore the unstable pole was

$$
s_{\theta 1}=\sqrt{\frac{\left(m_{b}+3 m_{w}\right) g}{\left(m_{b}+6 m_{w}\right) d}}
$$

The value of the pole is tabulated in table 2 as $\mathrm{d}$ change, $d \in[0.1,2]$.

Table 2.Variation of unstable pole over $d$.

\begin{tabular}{|l|l|l|l|l|l|l|}
\hline $\mathrm{d}(\mathrm{m})$ & 0.1 & 0.3 & 0.5 & 0.7 & 0.9 & 1.1 \\
\hline $\mathrm{S}_{\theta 1}$ & 9.542 & 5.509 & 4.267 & 3.606 & 3.181 & 2.877 \\
\hline
\end{tabular}

From the table 1, pole became larger when $\mathrm{d}$ was shorter. This matches the fact that the robot will be harder to balance when COG of the main body is lower as shown in Fig. 3. However for higher $\mathrm{d}$, larger torque will be required.

Proof: To hold the pendulum at vertical, defines the displacement of point $C, x_{C}$ must be equal to circumference, $C_{d}$, that had been made by $\theta$ at point $d$.

$$
x_{C} \equiv C_{d}=\theta d,
$$

Thus

$$
F_{C}=m_{b} a_{C}=\frac{m_{b} x_{C}}{t^{2}}=\frac{m_{b} \theta d}{t^{2}},
$$


It is proven by newton's law, larger force is required if $\mathrm{d}$ becomes larger.

\subsubsection{Effect of mass of body on tilt angle, $\theta$, control}

Table 3 below shows that when the mass of the body increase to $20 \mathrm{~kg}$ the unstable pole only increase about 1 from its initial value. Thus the effect is less significant on the tilt angle balancing control. This is true as shown in Fig. 4(a).

Table 3. Variation of unstable pole over $m_{b}$.

\begin{tabular}{|l|c|c|c|c|c|c|}
\hline $\mathrm{m}_{\mathrm{b}}(\mathrm{kg})$ & 1 & 2 & 4 & 6 & 10 & 20 \\
\hline $\mathrm{S}_{\theta 1}$ & 5.451 & 5.777 & 6.110 & 6.279 & 6.451 & 6.609 \\
\hline
\end{tabular}

\subsubsection{Effect of mass of wheel on tilt angle, $\theta$, control}

Table 4 shows that when the mass of wheel increase to $2 \mathrm{~kg}$ the unstable pole only decreases about 0.6 from its initial value. Thus the effect is less significant on the tilt angle balancing control as shown in Fig. 4(b).

Table 4. Variation of unstable pole over $m_{w}$.

\begin{tabular}{|l|c|c|c|c|c|c|}
\hline $\mathrm{m}_{\mathrm{w}}(\mathrm{kg})$ & 0.2 & 0.4 & 0.6 & 0.8 & 1 & 2 \\
\hline $\mathrm{S}_{\theta 1}$ & 6.675 & 6.564 & 6.465 & 6.377 & 6.298 & 5.999 \\
\hline
\end{tabular}

\subsubsection{Inertia forces over initial condition}

From the model, the motors need to generate enough amount of torque to compensate inertia forces, $F_{1}^{*}, F_{2}^{*}$, and $F_{3}^{*}$ while balancing and moving in the Cartesian plane. Thus these inertia forces were analysed over three initial conditions of position, $x$, tilt angle, $\theta$, and heading angle, $\phi$. Three initial conditions are considered, $(x, \theta, \phi)=(0.01745 \mathrm{~m}, 0,0),(x, \theta, \phi)=(0,0.01745 \mathrm{rad}, 0)$, and $(x, \theta, \phi)=(0,0,0.01745 \mathrm{rad})$.

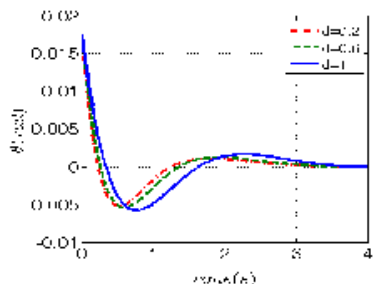

(a)

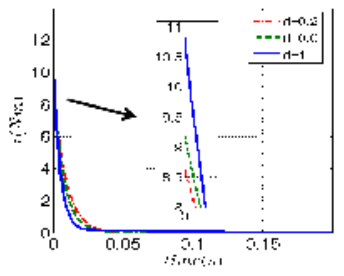

(b)

Figure 3. Responses of (a) tilt angle and (b) input torque over various COG of robot main body 


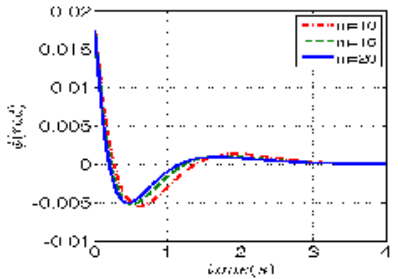

(a)

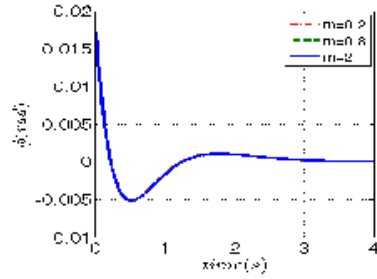

(b)

Figure 4. Response of (a) tilt angle of over various mass of body and (b) tilt angle of over various mass of wheel

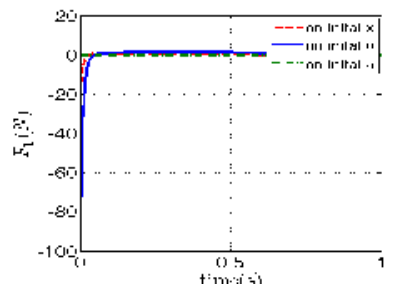

(a)

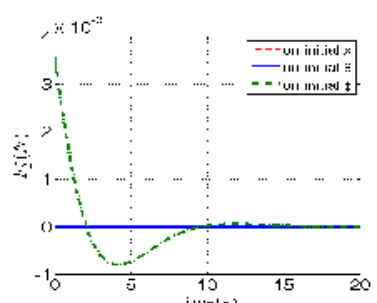

(b)

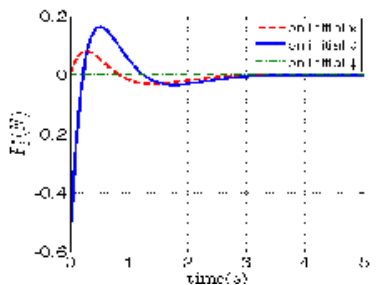

(c)

Figure 5. Responses of (a) Inertia force $F_{1}^{*}$, (b) Inertia force $F_{2}^{*}$, and (c) Inertia force $F_{3}^{*}$ due to $x, \theta$, and $\phi$ initial conditions.

Fig. 5 shows the effect of these three initial conditions. In this work, WIP's motors have maximum torque $\tau_{w r(\max )}=4 \mathrm{Nm}$. Thus it is important to determine the radius of the wheels. Based on force and torque relationship, the wheels radius $R$ must be less than the ratio of maximum wheels torque and maximum heading inertia force, $2 \tau_{w r(\max )} / F_{1(\max )}^{*}$. It was shown in Fig. 5(a), maximum heading inertia force $F_{1(\max )}^{*}$ is about $81.22 \mathrm{~N}$ when robot needs to compensate tilt angle initial error. Thus, based on this fact, R should less than $0.098 \mathrm{~m}$

\subsection{Controller analysis}

\subsubsection{Basic input test}

A WIP system with LQR controller tested on three types of input, unit step, ramp function, and exponential function. The test showed the suitability of input to the mobile WIP system application. Fig. 6 shows, LQR controller was the best on unit step input. However its performance deteriorated when operates on others input function as shown in Fig. 7 and Fig. 8. Fig. 7 shows, WIP with LQR had position and heading states error on ramp input. Fig. 8 shows, WIP with LQR had position, velocity, heading angle, heading angular velocity state errors occur in exponential input. For mobile WIP application, unit step input was not suitable where it required tremendous large amount of torque as maximum as $45 \mathrm{Nm}$ as showed in Fig. 6(c). In contrast, ramp and exponential type input functions are suitable input for the WIP system; these inputs do not only have velocity information, it also enables the WIP system to be driven in optimum velocity using permissible torque energy as shown in Fig. 7(c) and Fig. 8(c). 


\subsubsection{Cartesian motion}

WIP was tested on two types of motion, rectilinear and curvilinear motions. WIP needed to be held in a vertical while moving to a specify distance either in a straight line or in a curve. Below are the equations of position input and heading angle input for both rectilinear and curvilinear motions.

$$
\begin{aligned}
x_{i n}(t) & =\frac{P o s}{1+e^{-T\left(t-T_{c}\right)}}, \\
\phi_{i n}(t) & =\frac{-H e a}{1+e^{-T\left(t-T_{c}\right)}},
\end{aligned}
$$

The constants $\mathrm{T}=0.3, \mathrm{Tc}=20$ for both types of motions. The constants $P o s=5, H e a=0$ and $P o s=1.25 \pi, \mathrm{Hea}=\pi / 4$ were respectively for rectilinear and curvilinear motions. Both inputs have s-shape trajectory and its slope is maximum time $t=T_{c}$.

From both Fig. 9 and 10, system with PSPID controller had better dynamic performance over the system with LQR controller. Comparatively, WIP with PSPID controller took only 40s to reach at specified position and orientation compare to WIP with LQR controller which took twice time longer (about 80s) as shown in Fig. 10(e). It verified that the trajectory error can be reduced to zero by adding an additional pole at zero to the system; and this process was achievable by implementing PSPID controller to the WIP system. Moreover on QOR tuning formulation, required torque of the WIP system with PSPID can be guaranteed within permissible torque limitation.

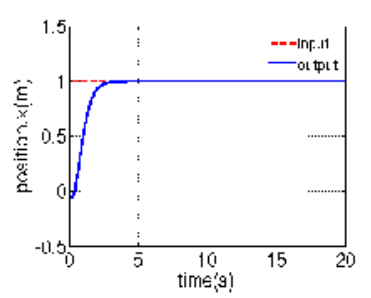

(a)

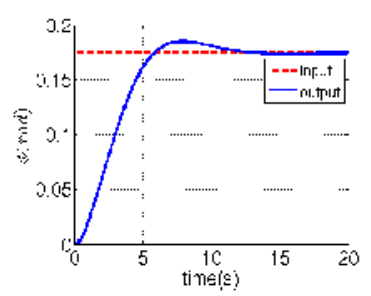

(b)

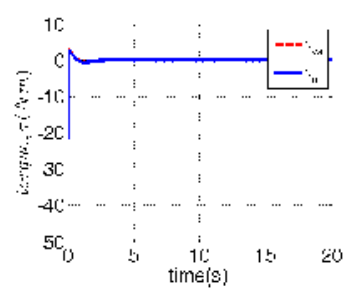

(c)

Figure 6. Responses of (a) state $x$, (b) state $\phi$ and (a) torques $\tau_{w r}, \tau_{w l}$ on unit step input.

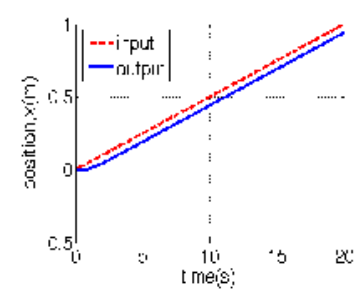

(a)

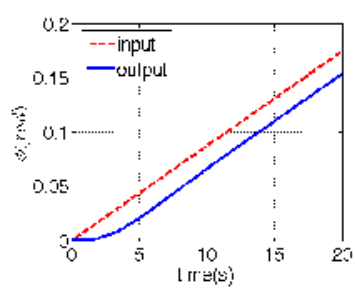

(b)

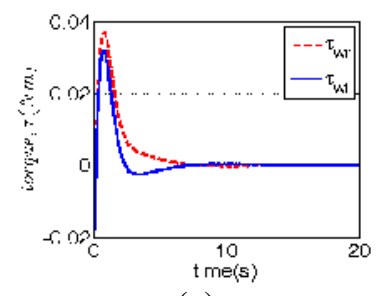

(c)

Figure 7. Responses of (a) state $x$, (b) state $\phi$ and (c) torques $\tau_{w r}, \tau_{w l}$ on ramp input. 
International Journal of Control Theory and Computer Modeling (IJCTCM) Vol.3, No.6, November 2013

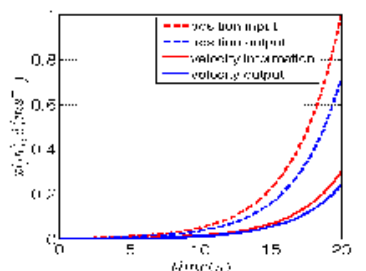

(a)

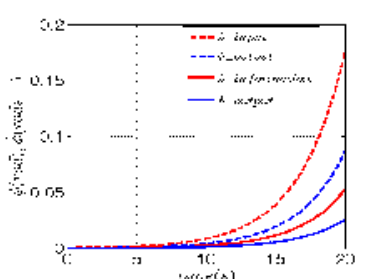

(b)

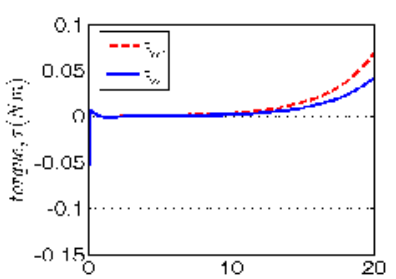

(c)

Figure 8. Responses of (a) states $x,{ }^{\dot{x}}$, (b) states $\phi, \dot{\phi}$ and (c) torques $\tau_{w r}, \tau_{w l}$ on exponential input.

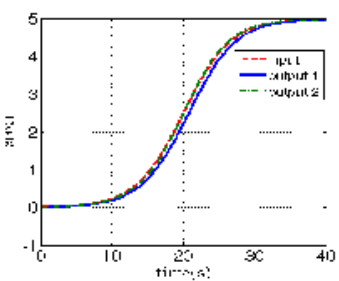

(a)

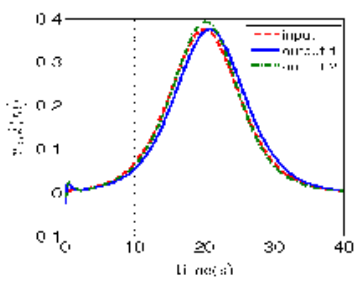

(b)

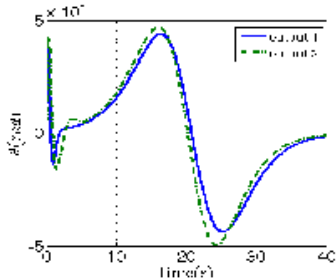

(c)

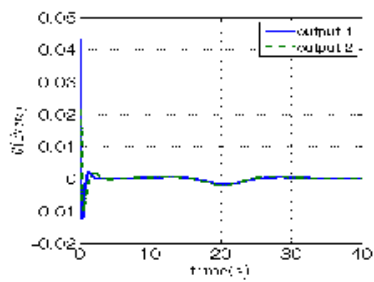

(d)

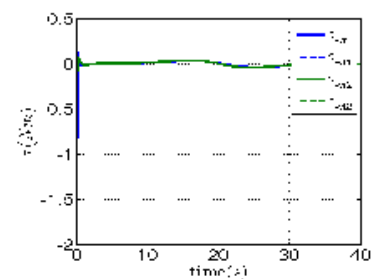

(e)

Figure 9. Responses of (a) state $x$, (b) state ${ }^{\dot{x}}$, (c) state $\theta$, (d) state $\dot{\theta}$ and (e) torques $\tau_{w r}, \tau_{w l}$ on rectilinear motion. 


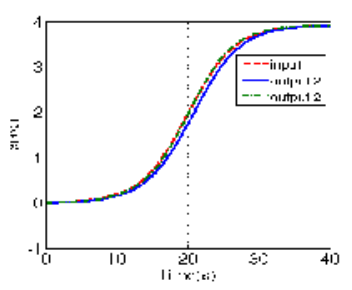

(a)

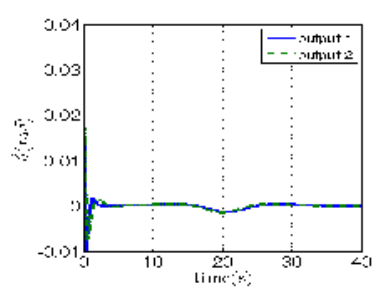

(d)

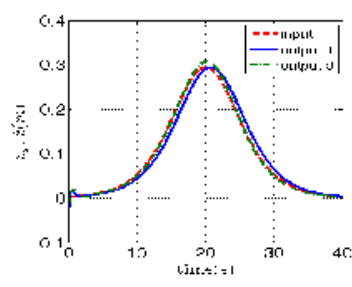

(b)

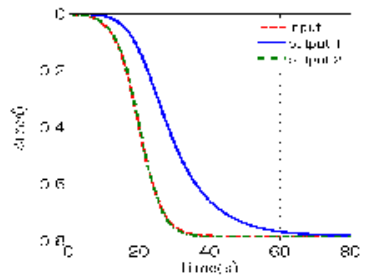

(e)

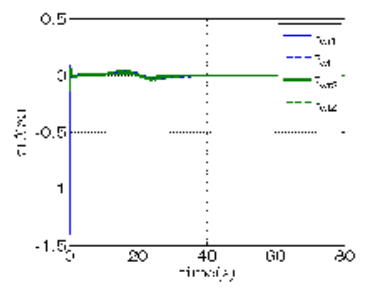

(g)

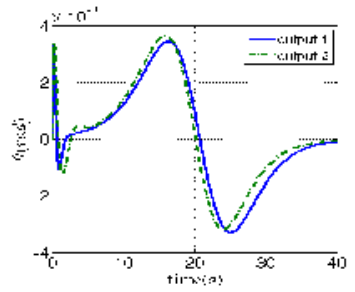

(c)

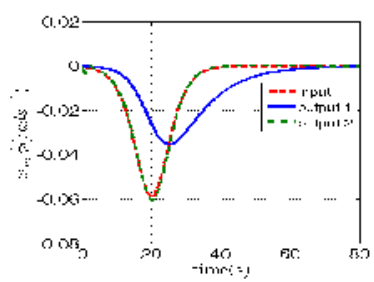

(f)

Figure 10. Responses of (a) state $x$, (b) state ${ }^{\dot{x}}$, (c) state $\theta$, (d) state $\dot{\theta}$, (e) state $\phi$, (f) state $\dot{\phi}$ and (g) torques $\tau_{w r}, \tau_{w l}$ on curvilinear motion.

\section{Conclusion}

Nonlinear dynamic mathematical model of a WIP system successfully derived. Applying right geometric context of the active force and inertia force led to a correct derivation of the WIP system using Kane's method. Furthermore, an optimal PSPID controller had been proposed to control the system for better dynamic performance. The controller had both advantages of PID and QOR tuning method.

Theoretical design, simulation and numerical results depicted that: 1) the nonlinear dynamic model correctly derived, 2) WIP system with proposed controller had a satisfactory dynamic performance where the trajectory error was very small, 3) QOR tuning scheme used and this guarantee stability of WIP system, and 4) WIP required low magnitude of torque and this permit practical implementation.

Further research should concern on: 1) variable type of WIP model since it is closer to human transporter and WIP service robot application and 2) design and implementation of the proposed controller in a microcontroller based system as a standalone and low level controller since of its simple and easy realization. 


\section{APPENDIX}

Nonlinear and linear WIP state variables

$$
\begin{aligned}
& D_{I P 1}=\left(m_{b}+3 m_{w}\right)\left(m_{b} d^{2}+I_{B 3}\right)-\left(m_{b} d \cos \theta\right)^{2}, \\
& D_{I P 2}=m_{b}(d \sin \theta)^{2}+I_{b 2}+\left(4 L^{2}+\frac{R^{2}}{2}+(L \cos \phi)^{2}\right) m_{w}, \\
& A_{24}=\frac{\left(m_{b} d^{2}+I_{b 3}\right) m_{b} d \sin \theta \dot{\theta}}{D_{I P 1}}, A_{26}=\frac{\left(m_{b} d^{2}\left(1-\cos ^{2} \theta\right)+I_{b 3}\right) m_{b} d \sin \theta \dot{\phi}}{D_{I P 1}}, \\
& A_{44}=\frac{-\left(m_{b} d\right)^{2} \sin \theta \cos \theta \dot{\theta}}{D_{I P 1}}, A_{46}=\frac{3 m_{w} m_{b} d^{2} \sin \theta \cos \theta \dot{\phi}}{D_{I P 1}}, A_{66}=\frac{-m_{b} d^{2} \sin \theta \cos \theta \dot{\theta}}{D_{I P 2}}, \\
& B_{21}=B_{22}=\frac{\left(m_{b} d^{2}+I_{b 3}\right)}{D_{I P 1} R}, B_{41}=B_{42}=\frac{-m_{b} d \cos \theta}{D_{I P 1} R}, B_{61}=-B_{61}=\frac{L \cos \phi}{D_{I P 2} R}, \\
& F_{2}=\frac{-\left(m_{b} d\right)^{2} g \sin \theta \cos \theta}{D_{I P}}, F_{4}=\frac{m_{b} d g \sin \theta\left(m_{b}+3 m_{w}\right)}{D_{I P}}, \\
& D_{I P L 1}=\left(m_{B}+3 m_{w}\right)\left(m_{b} d^{2}+I_{b 3}\right)-\left(m_{b} d\right)^{2}, \\
& D_{I P L 2}=I_{b 2}+\left(5 L^{2}+\frac{R^{2}}{2}\right) m_{w}, \\
& A_{23 L}=\frac{-\left(m_{b} d\right)^{2} g}{D_{I P L 1}}, A_{43 L}=\frac{\left(m_{b}+3 m_{w}\right) m_{b} d g}{D_{I P L 1}}, \\
& B_{21 L}=B_{22 L}=\frac{\left(m_{b} d^{2}+I_{B 3}\right)}{D_{I P L 1} R}, B_{41 L}=B_{42 L}=\frac{-m_{b} d}{D_{I P L 1} R}, B_{61 L}=-B_{62 L}=\frac{L}{D_{I P L 2} R},
\end{aligned}
$$

\section{REFERENCES}

[1] Z. Kausar, K. Stol, and N. Patel, "The Effect of Terrain Inclination on Performance and the Stability Region of Two-Wheeled Mobile Robots," International Journal of Advanced Robotic Systems, vol. 9, no. 218, pp. 1-11, 2012.

[2] S. H. Miao, "Modeling of self-tilt-up motion for a two-wheeled inverted pendulum," Industrial Robotan International Journal, vol. 38, no. 1, pp. 76-85, 2011.

[3] F. Grasser, A. D’Arrigo, S. Colombi, and A. C. Rufer, "JOE: a mobile, inverted pendulum," IEEE Transactions on Industrial Electronics, vol. 49, no. 1, pp. 107-114, 2002.

[4] L. Jingtao, X. Gao, H. Qiang, D. Qinjun, and D. Xingguang, "Mechanical Design and Dynamic Modeling of a Two-Wheeled Inverted Pendulum Mobile Robot," Automation and Logistics, 2007 IEEE International Conference on, Jinan, China, pp. 1614-1619, 2007.

[5] M. Baloh and M. Parent, "Modeling and Model Verification of an Intelligent Self-Balancing TwoWheeled Vehicle for an Autonomous Urban Transportation System," Conference on Computational Intelligence, Robotics, and Autonomous Systems, Singapore, pp. 1-7, 2003.

[6] J. S. Noh, G. H. Lee, and S. Jung, "Position control of a mobile inverted pendulum system using radial basis function network," International Journal of Control, Automation and Systems, vol. 8, no. 1, pp. 157-162, 2010

[7] H. Ozaki, T. Ohgushi, T. Shimogawa, and C.-J. Lin, "Position and orientation control of a wheeled inverted pendulum," JSME International Journal, Series C: Mechanical Systems, Machine Elements and Manufacturing, vol. 44, no. 1, pp. 188-195, 2001.

[8] K. Teeyapan, J. Wang, T. Kunz, and M. Stilman, "Robot Limbo: Optimized Planning and Control for Dynamically Stable Robots Under Vertical Obstacles," 2010 IEEE International Conference on Robotics and Automation, Anchorage, Alaska, USA, pp. 4519-45 S. H. Jeong and T. Takahashi, "Wheeled inverted pendulum type assistant robot: inverted mobile, standing, and sitting motions," 
IEEE/RSJ International Conference on Intelligent Robots and Systems 2007, San Diego, CA, USA., pp. 1932-1937, 2007.

[10] M. L. Chen, "Analysis and design of robust feedback control systems for a nonlinear two-wheel inverted pendulum system," 2012 International Symposium on Computer, Consumer and Control, Taichung City, Taiwan, pp. 949-953, 2012.

[11] K. M. Goher, M. O. Tokhi, and N. H. Siddique, "Dynamic modelling and control of a two wheeled robotic vehicle with a virtual payload," Journal of Engineering and Applied Sciences, vol. 6, no. 3, pp. 7-41, 2011.

[12] A. Bajodah, D. Hodges, and Y.-H. Chen, "Nonminimal Kane's Equations of Motion for Multibody Dynamical Systems Subject to Nonlinear Nonholonomic Constraints," Multibody System Dynamics, vol. 14, no. 2, pp. 155-187, 2005.

[13] Y. Kim, S. Kim, and Y. Kwak, "Dynamic analysis of a nonholonomic two-wheeled inverted pendulum robot," Journal of Intelligent and Robotic Systems, vol. 44, no. 1, pp. 25-46, 2005.

[14] H. Jian, G. Zhi-Hong, T. Matsuno, T. Fukuda, and K. Sekiyama, "Sliding-Mode Velocity Control of Mobile-Wheeled Inverted-Pendulum Systems," Robotics, IEEE Transactions on, vol. 26, no. 4, pp. 750-758, 2010.

[15] C. H. Chiu and C. H. Lin, "A WIP control based on an intelligent controller," World Academy Of Science Engineering and Technology vol. 54, no. 126, pp. 656-661, 2011.

[16] C. H. Huang, W. J. Wang, and C. H. Chiu, "Design and implementation of fuzzy control on a twowheel inverted pendulum," IEEE Transactions on Industrial Electronics, vol. 58, no. 7, pp. 29883001, 2011.

[17] C. C. Tsai, H. C. Huang, and S. C. Lin, "Adaptive Neural Network Control of a Self-Balancing TwoWheeled Scooter," Industrial Electronics, IEEE Transactions on, vol. 57, no. 4, pp. 1420-1428, 2010.

[18] E. Minnaert, B. Hemmelman, and D. Dolan, "Inverted pendulum design with hardware fuzzy logic controller," 11th World Multi-Conference on Systemics, Cybernetics and Informatics, (Wmsci 2007), Orlando, pp. 76-81, 2007.

[19] T. Ren, T. C. Chen, and C. J. Chen, "Motion control for a two-wheeled vehicle using a self-tuning PID controller," Control Engineering Practice, vol. 16, no. 3, pp. 365-375, 2008.

[20] M. Fallahi and S. Azadi, "Adaptive Control of an inverted pendulum using adaptive PID neural network," Proceedings of the 2009 International Conference on Signal Processing Systems, Los Alamitos, CA, USA, pp. 589-593, 2009.

[21] S. Liang and G. Jiafei, "Researching of Two-Wheeled Self-Balancing Robot Base on LQR Combined with PID," 2nd International Workshop on Intelligent Systems and Applications (ISA), pp. 1-5, 2010.

[22] A. N. K. Nasir, M. A. Ahmad, and R. M. T. R. Ismail, "The control of a highly nonlinear two-wheels balancing robot: A comparative assessment between LQR and PID-PID control schemes," World Academy Of Science Engineering and Technology, vol. 46, no. 44, pp. 227-232, 2010.

[23] S. Ahmad and M. O. Tokhi, "Linear Quadratic Regulator (LQR) approach for lifting and stabilizing of two-wheeled wheelchair," 4th International Conference On Mechatronics (ICOM), pp. 1-6, 2011.

[24] S. Y. Seo, S. H. Kim, S.-H. Lee, S. H. Han, and H. S. Kim, "Simulation of attitude control of a wheeled inverted pendulum," International Conference on Control, Automation and Systems, (ICCAS '07), pp. 2264-2269, 2007.

[25] T. Takei, R. Imamura, and S. Yuta, "Baggage Transportation and Navigation by a Wheeled Inverted Pendulum Mobile Robot," IEEE Transactions on Industrial Electronics, vol. 56, no. 10, pp. 3985 3994, 2009. 


\section{Authors}

Mohamad Amir Shamsudin was born in Johor, Malaysia, in 1983. He received the B.S and M.S degrees in mechatronics engineering from Universiti Teknologi Malaysia (UTM) respectively in 2007 and 2009. He is currently a PhD student in the Faculty of Electrical Engineering, UTM. His research is on Modelling and Control of Wheel Inverted Pendulum system.

Rosbi Mamat is Associate Professor at Department of Control and Mechatronic UTM, Malaysia. His research interests include control and intelligent systems, mechatronic system, and embedded system.

Sophan Wahyudi Nawawi received his PhD from UTM, Malaysia in 2006. His PhD research was in Control area. His research interests include nonlinear system and control, robust control and robotic system.
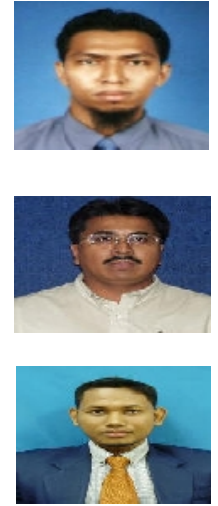